\title{
Relationship Between Clinical Learning and Classroom Learning with the Perspective of Confidence Level on Patient Safety Among Baccalaureate Nursing Students
}

\author{
Tayyba Nazar Muhammad Hussain Muhammad Afzal Syed Amir Gilani \\ The University of Lahore
}

\begin{abstract}
Patient safety is a global issue in these days. Nurses play important role in patient safety through monitoring the patient in front line of health care team. The international nursing group and global health were growing the essential ability to improve patient safety. To make nurses more confident in patient safety, early training with protection of affected person as a prominent feature of the nursing curriculum is necessary. The objective of study to check the Relationship between Clinical Learning and Classroom Learning with the perspective of Confidence level on Patient Safety among Nursing Students. . Descriptive cross sectional study conducted in the Lahore school of nursing, the University of Lahore. The study data collected using a questionnaire, Health professional Education in Patient Safety Survey (HPESS), which consisted on 7 subscales. The target population 170 and the study was conducted on Baccaular of Science in Nursing (Hones) and Baccaular of Science in Nursing (Post RN) students. The results of this study have given the picture of the association of classroom learning with that of the implementation of the clinical practice. By incorporating the higher level of objectives about patient safety in objection in learning in nursing curriculum, students will be able to gain more knowledge about the importance of patient safety issue, which will increase the confidence level in classroom and clinical learning on patient safety among nursing students.
\end{abstract}

Keywords: Patient Safety, Nursing Curriculum, Confidence Level.

DOI: $10.7176 / \mathrm{JHMN} / 62-04$

Publication date:May $31^{\text {st }} 2019$

\section{Introduction}

To make safer Health Care System, patient safety has been known as global issue with increasing of preventable errors in health. Nurses are the prominent member of health care team; they play important role in patient safety through monitoring the patient in front line of health care team. To make nurses more competent in patient safety, early training with protection of affected person as a prominent feature of the nursing curriculum is necessary. Nursing school has adequate education about patient safety. Nursing schools know the need for educational innovations to teach patient safety. (Lee, Jang, \& Park, 2016).

Hand hygiene is a basis in the prevention of hospital acquired infection and clinical safety. It has been noted that at least $25 \%$ of all infections of hospitalized patients in the developing world are health care associate. It is also notified that hand hygiene is the part of clinical safety. Studies have shown that hand hygiene compliance rates in developed and developing countries rarely exceeded 50\%. The World Health Organization recommends 5 moments of hand hygiene during health care delivery as essential for safe patient care.(Niyonzima, Brennaman, \& Beinempaka, 2018).

Patient safety is most often associated with physical and procedural elements as it relates to avoiding, 
eliminating or removing adverse events. Culture shapes all elements of a person's or a group's life. Each culture has its own personal traits that define the normative values of its members. From another perspective culture have an impact on nurses clinical practice and confidence level that could range from another subculture. In Asian countries nurses followed human limitation rule, in which basic needs perform by family members, on the other hand Western countries people totally depend upon nurses to meet their health task. In clinical settings, cultural difference and language barrier can effect quality care, confidence level and patient safety.(Almutairi, Gardner, \& McCarthy, 2013).

Nurse's teamwork is an important part of quality health care and patient safety. Nurses and patients both were benefited by teamwork, nurses can gain self-retention, job satisfaction and increase in expertise of nursing staff while in patient teamwork decrease mortality rate, decrease errors and adverse events and increase patient safety. Nursing students have proper attention by teamwork, which helps them increase their knowledge and skills. (Polis, Higgs, Manning, Netto, \& Fernandez, 2017)

Proper communication between nurses and patients is important for the successful outcome of nursing care of every patient. Communication between health care members related to patient care express sincere concern with patients. Effective non-verbal communication reduce adverse events in nursing practice.(Kourkouta \& Papathanasiou, 2014).

WHO has identified that patient safety is a global issue, through all over the world. Millions of peoples suffer with disease and deaths due to unsafe medical care. Most adverse factors occur due to different preceptors of health care team human factor errors(negligence, lack of attention) and system factor errors(resources, educational system).(Pelzang \& Hutchinson, 2018)

Comfort speaking is very important for patient safety, the nurses who provide higher levels of patient care to take personal risks and are more willing to speak up about patient safety concerns. Both management-based and commitment-based approaches seem to be relevant for managing patient safety. (Alingh, van Wijngaarden, van de Voorde, Paauwe, \& Huijsman, 2019)

This study conducted in Hospital of Pakistan to find out the knowledge of patient safety among postgraduate residents (PGR) and nurses. Unfortunately medical and nursing having no curriculum about patient safety in Pakistan. The postgraduate residents in the first two years of their study having high (67\%) positivity about patient safety culture, then the students who have completed their graduation had lowest positivity (53\%) about patient safety. Nursing students having (65\%) about perception of patient safety culture. so both postgraduate students and nursing student's perception showed $80 \%$ to $87 \%$ required education about patient safety in undergraduate students.(Bari, Jabeen, Bano, \& Rathore, 2017)

According to this study, Patient safety is the basic root of greatest health care system. There is highest favorable responses were attained on overall practices of patient safety $(75 \%)$, and the adverse events which actually harmed the patients (67\%). On the other hand, $80 \%$ nurses expressed their concern for personal errors in work. Similarly, $52 \%$ Indicated inhibitions on communication openness and only $32 \%$ endorsed the provision of feedback and communication about errors.(Zubair, Kamal, \& Razik, 2017)

Significance of the study: This study explored the confidence level in nursing students is low in clinical learning then classroom learning. By making the part of patient safety in nursing curriculum, students will be able to gain more knowledge about the importance of patient safety issue, which will prevent harms. These evaluations of 
effect of confidence level on patient safety in nursing students. It is the window for others researchers for research on this topic regarding the confidence level effects on patient safety among nursing students.

\section{Theoretical Framework:}

Theoretical framework employed in this research work will be shown, according the interconnections between the constructs of confidence, and patient safety are best described within the Bandura's Social Cognitive Theory. Bandura Described self-efficacy as the "belief one has in being able to execute a specific task successfully. I use the term self-confidence instead of self-efficacy because it is more familiar to most educators. Self-confidence influences virtually every aspect of an individual's life, from the individual's ability to think optimistically, persevere through difficulties, and ultimately, completes activities in their clinical factor, environmental factor and personal factors

\section{Materials and Methods}

A quantitative cross-sectional research design was used for this study

\section{Independent variables}

Confidence level

\section{Dependent Variable}

Patient safety

\section{Sample Size \& Sampling Techniques:}

Data was collected from the participant through questionnaire and the participants were selected through convenient sampling method, the sample size for this study were 170 nurses students which is calculated from the Slovene's formula.

Inclusion Criteria: Following students from school of nursing in University of Lahore were included in data collection, those who were present at the time of data collection, those who were willing to participate and students, who were 16-29 years old

Exclusion Criteria: Following students from school of nursing in University of Lahore will be not included in data collection; students are not present in this study, those who were not present at the time of data collection, those who were not willing to participate and Students, who were less than 16 year and more than30 years old. 


\section{Result and Discussions:}

Table1: Mean of the Health Professional Education in Patient Safety survey subscales among Student Nurses in Classroom and Clinical

\begin{tabular}{|c|c|c|c|}
\hline \multirow[t]{2}{*}{ S.No } & \multirow[t]{2}{*}{ Question } & \multicolumn{2}{|c|}{ Mean } \\
\hline & & Classroom & Clinical \\
\hline \multirow[t]{8}{*}{1} & Section 1: Learning about specific patient safety content areas & & \\
\hline & 1. Clinical safety: I am confident what I learned about: & 2.8 & 2.8 \\
\hline & 2. Culture of safety: I am confident what I learned about: & 3.14 & 3.12 \\
\hline & $\begin{array}{l}\text { 3. Working In Teams with Other Health Professionals: I am confident what I } \\
\text { learned about: }\end{array}$ & 3.27 & 3.23 \\
\hline & 4. Communicating Effectively: I am confident what I learned about: & 3.14 & 3.16 \\
\hline & 5. Managing Safety Risks: I am confident what I learned about: & 3.1 & 3.3 \\
\hline & $\begin{array}{l}\text { 6. Understanding Human and Environmental Factors: I am confident what I } \\
\text { learned about: }\end{array}$ & 3.1 & 3.13 \\
\hline & $\begin{array}{l}\text { 7. Recognize, Respond to and Disclose Adverse Events and Close Calls: I am } \\
\text { confident what I learned about: }\end{array}$ & 3.2 & 3.1 \\
\hline 2. & $\begin{array}{l}\text { Section } 2 \text { How broader patient safety issues are addressed in health } \\
\text { professional education. }\end{array}$ & 3.1 & 3.2 \\
\hline \multirow[t]{2}{*}{3.} & Section 3 Comfort speaking up about patient safety. & 3.1 & 3.3 \\
\hline & Total & 3.15 & 3.14 \\
\hline
\end{tabular}

Table 1 presents the mean of the health professional education in patient safety survey subscales among student nurses in classroom and clinical. The mean of the section 1 component of clinical safety it is 2.8 in clinical and in classroom. Moreover the score of culture safety in classroom and clinical were 3.14 and 3.12 respectively, which are almost similar. The section 3 regarding comfort speaking up about patient safety were also similar.

Table 02: Normality test of the Health Professional Education in Patient Safety Survey Scale among Student Nurses in Classroom and Clinical

S.No Health Professional Education in Patient Safety Survey Kolmogorov Smirnov Test Shapiro-Wilk

\begin{tabular}{llll}
\hline $\mathbf{1}$ & Classroom & 0.34 & 0.4 \\
$\mathbf{2}$ & Clinical & 0.28 & 0.35
\end{tabular}

Table 2 shows the normality test of the scales. This test was to fulfill the assumptions of normality. The table represents Komologorov Smirnov test of health professional education in patient safety survey scale among student nurses in classroom and clinical were 0.3 and 0.2 which were not significant and which represent that the data is normally distributed. 
Table 3: Internal Consistency of the Health Professional Education in Patient Safety Survey Scale among Student Nurses in Classroom and Clinical

\begin{tabular}{|lll|}
\hline S.No & Health Professional Education in Patient Safety Survey & Cronbach Alpha \\
\hline $\mathbf{1}$ & Classroom & 0.997 \\
\hline $\mathbf{2}$ & Clinical & 0.997 \\
\hline
\end{tabular}

Cronbach's alpha is a measure of internal consistency. This represents that how closely related is a set of items are as a group. Table 6 shows the cronbach alpha of the scales. This test was conducted to observe the reliability of the items of the scale in the population. Cronbach alpha of the health professional education in patient safety survey scale among student nurses in classroom and clinical were 0.99 and 0.99 respectively. This represents that the Cronbach alpha of the scales among the participants is good. Hence, these scale are appropriate to collect data in this population.

Conclusion The results of the study show that clinical performance and professional self-concept are related. Accordingly, attempts should be made to enhance students' professional self-concept during their education that will enhance students' clinical performance. These findings can be helpful to teachers, authorities at nursing colleges, and nursing managers.

\section{Discussion}

The objective of the article is to determine the relationship between perspectives of confidence level in classroom and clinical learning on patient safety among baccalaureate nursing students in the private university. The findings of the study showed positive relationship between confidence levels of students at the clinical and classroom levels, which shows that if students are learning the concepts in the classroom then they are applying that concept in the clinical. Many other studies also shared that there is a strong positive relationship between classroom learning and improved clinical practices (Ellis, 2016; Koivisto, Multisilta, Niemi, Katajisto, \& Eriksson, 2016; Slimmer, Wendt, \& Martinkus, 2016).

In the present study there was a strong positive relationship between the hand hygiene and infection control practices in class to the confidence of applying that in the clinical areas which is also supported by studies conducted elsewhere (Cruz \& Bashtawi, 2016; Stombaugh \& Judd 2014).

\section{Conclusion:}

The results of this study have given the picture of the association of classroom learning with that of the implementation of the clinical practice. The findings suggest nursing students are confident in what they are learning about clinical aspects of patient safety, however, their confidence in learning about sociocultural aspects declines as they are increasingly exposed to the clinical environment. This suggests a need to address the impact of the practice environment on nursing students' confidence in what they are learning about patient safety. There is direct effect of institutional and students learning with the class room learning to the clinical learning. By incorporating the higher level of objectives about patient safety in objection in learning in nursing curriculum, students will be able to gain more knowledge about the importance of patient safety issue which will increase the 
confidence level in classroom and clinical learning on patient safety among nursing students.

\section{References:}

01. Lee, N. J., Jang, H., \& Park, S. Y. (2016). Patient safety education and baccalaureate nursing students' patient safety competency: A cross-sectional study. Nursing \& health sciences, 18(2), 163-171.

02. Niyonzima, V., Brennaman, L., \& Beinempaka, F. (2018). Practice and compliance of essential handwashing among healthcare workers at a regional referral hospital in Uganda: A quality improvement and evidencebased practice. Canadian Journal of Infection Control, 33(1), 33-38.

03. Almutairi, A. F., Gardner, G., \& McCarthy, A. (2013). Perceptions of clinical safety climate of the multicultural nursing workforce in Saudi Arabia: A cross-sectional survey. Collegian, 20(3), 187-194.

04. Polis, S., Higgs, M., Manning, V., Netto, G., \& Fernandez, R. (2017). Factors contributing to nursing team work in an acute care tertiary hospital. Collegian, 24(1), 19-25.

05. Pelzang, R., \& Hutchinson, A. M. (2018). Patient safety issues and concerns in Bhutan's healthcare system: a qualitative exploratory descriptive study. BMJ open, 8(7), e022788.

06. Alingh, C. W., van Wijngaarden, J. D., van de Voorde, K., Paauwe, J., \& Huijsman, R. (2019). Speaking up about patient safety concerns: the influence of safety management approaches and climate on nurses' willingness to speak up. BMJ Qual Saf, 28(1), 39-48.

07. Bari, A., Jabeen, U., Bano, I., \& Rathore, A. W. (2017). Patient safety awareness among postgraduate students and nurses in a tertiary health care facility. Pakistan journal of medical sciences, 33(5), 1059.

08. Zubair, A., Kamal, A., \& Razik, S. (2017). Patient safety practices among nurses-perspectives from Islamabad and Rawalpindi. JPMA. The Journal of the Pakistan Medical Association, 67(8), 1304-1304.

09. Ellis, D. M. (2016). The role of nurse educators' self-perception and beliefs in the use of learner-centered teaching in the classroom. Nurse Education in Practice, 16(1), 66-70.

10. Koivisto, J. M., Multisilta, J., Niemi, H., Katajisto, J., \& Eriksson, E. (2016). Learning by playing: A crosssectional descriptive study of nursing students' experiences of learning clinical reasoning. Nurse education today, 45, 22-28.

11. Slimmer, L. W., Wendt, A., \& Martinkus, D. (2016). Effect of psychiatric clinical learning site on nursing students' attitudes toward mental illness and psychiatric nursing. Journal of Nursing Education, 29(3), $127-$ 133.

12. Cruz, J. P., \& Bashtawi, M. A. (2016). Predictors of hand hygiene practice among Saudi nursing students: A cross-sectional self-reported study. Journal of infection and public health, 9(4), 485-493.

13. Stombaugh, A., \& Judd, A. (2014). Does nursing assistant certification increase nursing student's confidence 
level of basic nursing care when entering a nursing program?. Journal of Professional Nursing, 30(2), 162167. 